\title{
Umwandlung wissenschaftlicher Journale in Gold Open Access
}

\author{
Chance oder Risiko für die Wissenschaft?
}

Heidemarie Hanekop, SOFI Göttingen, Friedländer Weg 31, 37085 Göttingen (heidi.hanekop@sofi.uni-goettingen.de)

Das Internet weckte Erwartungen auf offenen und kostenfreien Zugang zu den neuesten Erkenntnissen aus der Wissenschaft, wie ihn die OpenAccess-Bewegung seit 15 Jahren fordert. Bis heute sind ein Drittel der Aufsätze in Open-Access-Archiven oder OA-Journalen verfügbar. Die Marktposition der großen Verlage ist stärker als zuvor. Wissenschaftsallianzen wollen Open Access jetzt in Lizenzverhandlungen mit den Verlagen durch eine generelle Umwandlung des Geschäftsmodells durchsetzen. Statt des Zugangs soll künftig die Publikation kostenpflichtig sein. Der Beitrag diskutiert mögliche Risiken für die offene, ungehinderte Publikation neuer wissenschaftlicher Erkenntnisse. Gegenwärtig werden drei Ansätze verfolgt: Erstens, finanzielle Hürden für die Publikationschancen von AutorInnen werden durch pauschale Zahlung der Publikationsgebühren vermieden. Zweitens, das Risiko wird durch die Wahlmöglichkeit zwischen OA oder kostenfreier Publikation zunächst umgangen - mit Risiken für die Umwandlungsstrategie. Drittens, individuelle Publikationsgebühren für Autorlnnen werden durch Arbeitgeber oder Förderer erstattet - mit Risiken für Publikationsmöglichkeiten, die empirisch untersucht werden sollten.

\section{Transition of scientific journals to Gold Open Access \\ Opportunity or risk for science?}

The advent of the Internet gave rise to the expectation of open access to scientific knowledge and latest findings, which has been called for by the open access movement for the past 15 years. As yet, only one-third of scientific articles are available in open access archives or Open Access journals. Major publishers are in a stronger market position than ever. Alliances of scientific organizations now seek to achieve open access in licensing negotiations with publishers by overhauling the business model. Rather than charging for access, fees should be paid for publication. The article looks at potential risks to the open, unfettered publication of new scientific findings. Currently, three approaches are being pursued: (1) charging research institutions a flat publication fee to remove the burden from authors, (2) giving authors a choice between paid open access publication and free publication without open access, and (3) having the fees paid by au-

This is an article distributed under the terms of the Creative Commons Attribution License CCBY 4.0 (https://creativecommons.org/licenses/by/4.0/)

https://doi.org/10.14512/tatup.26.1-2.49

Eingereicht: 15. 03.2017. Angenommen: 01.06.2017 thors and reimbursed by their institutions - which may imperil open and unrestricted publication, an effect that must be empirically analyzed.

KEYWORDS: gold open access, scholarly communication, sociology of science

\section{Einführung}

Open Access (OA) bezeichnet den offenen, kostenfreien Zugang zu Forschungsergebnissen über das Internet. WissenschaftlerInnen haben vor mehr als zwei Jahrzehnten begonnen, die neuen technischen Möglichkeiten des Internets für den raschen, kostenfreien Austausch ihrer Ergebnisse zu nutzen, z. B. in dem 1991 gegründeten selbstorganisierten ArXiv, einem der großen, fachspezifischen Repositorien. Der Begriff Open Access wurde 2002 durch die Budapest Open Access Initiative und die Berliner Open-Access-Konferenz 2003 geprägt. Als Alternative zu den kostenpflichtigen wissenschaftlichen Zeitschriften der Verlage wurden zwei Open-Access-Strategien diskutiert, die Green Road basierend auf selbstorganisierten Repositorien (z. B. das arXiv), die Gold Road mit einem neuen Typ von Open-AccessJournalen (z. B. Public Library of Science, PLOS). Gleichzeitig hatten auch die marktführenden kommerziellen Verlage wie Elsevier und Springer oder Universitätsverlage bzw. Verlage wissenschaftlicher Gesellschaften (scholarly publisher) wie Oxford University Press, die Internettechnologien adaptiert, ihre Journale digitalisiert und als eJournale in die lizenzbasierten Geschäftsmodelle integriert. Vierzehn Jahre später, auf der 13. Berlin Konferenz 2017 diskutierte man nicht mehr über Alternativen zu den Journalen der marktführenden Verlage, sondern über deren Umwandlung in Gold-Open-Access-Journale. Dies beleuchtet schlaglichtartig zum einen die Stabilität des traditionellen wissenschaftlichen Publikationssystems. Denn die Digitalisierung wissenschaftlicher Journale hat zwar deren äußere Form verändert, aber die marktführenden Verlage sind gestärkt daraus hervorgegangen. Über 50 \% der Aufsätze entfallen 2013 auf Journale der fünf größten Verlage, 1996 hatte ihr Anteil noch 
bei $30 \%$ gelegen, 1973 bei $20 \%$ (Larivière et al. 2015, S. 3). Die durch die Wissenschaftsorganisationen ${ }^{1}$ aufzubringenden Lizenzkosten sind stark gestiegen und beschneiden die Mittel für die Wissensproduktion (Schimmer et al. 2015). Zum anderen zeigt die Diskussion auf der 13. Berlin Konferenz den Wandel der Open-Access-Bewegung. Die Wissenschaftsorganisationen wollen mit einer neuen Strategie den kostenfreien Zugang zu Journalen in den nächsten Jahren durch die umfassende Umwandlung in Open-Access-Journale erreichen (oa2020 2016).

In dem folgenden Beitrag sollen Chancen und Risiken der neuen Strategie vor dem Hintergrund der bisherigen Open-Access-Entwicklung diskutiert werden. Im Mittelpunkt steht dabei

\section{Das Spannungsverhältnis zwischen öffentlicher Wissensproduktion und Wertschöpfung der Verlage}

Im öffentlich finanzierten Wissenschaftssystem wird neues Wissen als öffentliches Gut geschaffen. Es zeichnet sich dadurch aus, dass es neu, relevant und ausschließlich der wissenschaftlichen Wahrheit verpflichtet sein soll, weder Religion, Herkunft noch Geld sollen den wissenschaftlichen Erkenntnisprozess stören, beeinflussen oder bestimmen. Die Wissensproduktion folgt gemeinschaftlichen Regeln und Normen (Merton 1985; Gläser 2006), die den uneingeschränkten Austausch des neuen Wissens

\section{Führt die generelle Transformation des Wertschöpfungs- modells von Lizenz- zu Publikationsgebühren zur Beschränkung der Publikationschancen von WissenschaftlerInnen?}

die Frage, was die generelle Einführung von Open-Access-Publikationsgebühren für AutorInnen bedeuten würde. Denn aus wissenschaftssoziologischer Sicht wirft die Transformation des Wertschöpfungsmodells von Lizenz- zu Publikationsgebühren Fragen auf, z. B. wie diese von den AutorInnen aufgebracht werden sollen? Werden sie zur Einschränkung von Publikationschancen und zur Benachteiligung von AutorInnen aus finanzschwachen Forschungseinrichtungen führen (Hofmann 2014; Simukovic 2016)? Treffen solche Befürchtungen zu, könnte sich die Beteiligung von AutorInnen als voraussetzungsvoll erweisen und ihr Publikationsverhalten zu unintendierten Effekten führen, die möglicherweise den Erfolg der Open-Access-Strategie gefährden. Dies wäre insbesondere in den Fächern zu erwarten, in denen Publikationsgebühren bisher kaum in der Publikationskultur verankert sind (Taubert 2014, 2017). Da der Erfolg der Strategie auf die aktive Beteiligung der WissenschaftlerInnen als Autoren, Editoren und Gutachter angewiesen ist (Meijer 2017), könnte sich der Umgang mit Open-Access-Publikationsgebühren in der Wissenschaft als eine entscheidende Schwachstelle erweisen, wenn solche Risiken nicht beachtet und vermieden werden. Dazu sollen die folgenden Überlegungen beitragen. Die Argumentation erfolgt in drei Schritten. Im ersten Abschnitt wird der wissenschaftssoziologische Bezugsrahmen skizziert. Danach folgt ein Überblick über die bisherige Verbreitung von Open Access. Im dritten Abschnitt wird die neue Open-Access-Strategie im Hinblick auf ihre Risiken für AutorInnen diskutiert, um auf kritische Punkte und möglicherweise unintendierte Effekte des Verhaltens von AutorInnen aufmerksam zu machen.

1 Als Wissenschaftsorganisationen bezeichne ich im Folgenden alle Einrichtungen, die öffentliche Gelder für die akademische Wissensproduktion erhalten, also Forschungseinrichtungen, Universitäten und die öffentlichen Einrichtungen der Wissenschaftsförderung, wie die DFG, nicht jedoch private oder gemeinnützige Stiftungen. zwischen WissenschaftlerInnen fördern, die Produktion dieser Art von Wissen stimulieren und seine private Aneignung verhindern sollen. Dem dient auch das (nicht monetäre) Belohnungssystem in Form von wissenschaftlicher Anerkennung durch Zitation. Es beruht auf einer besonderen Form der Qualitätssicherung (peer review), die nur solche Forschungsergebnisse zur Publikation zulässt, die wissenschaftlichen Kriterien entsprechen. Wissenschaftliche Journale sind, insbesondere in den Fächern der Natur-, Ingenieur- und Lebenswissenschaften, die Institution des Wissenschaftssystems, die die Funktionen der Qualitätsprüfung, Bewertung und Verbreitung gewährleisten sollen.

Wissenschaftliche Journale sind gleichzeitig Grundlage der Wertschöpfung von wissenschaftlichen Verlagen. Sie erstellen (u. a.) Journale und verkaufen sie als Ware auf einem Markt, dessen hauptsächliche Nachfrager wissenschaftliche Bibliotheken sind (Kommodifizierung wissenschaftlichen Wissens). Neben der Verbreitung ist eine zentrale Aufgabe von Verlagen die Organisation der Qualitätssicherung und Bewertung, die allerdings von WissenschaftlerInnen als Editoren und Gutachtern meist unentgeltlich im Auftrag der Verlage durchgeführt wird. Diese Verknüpfung von wissenschaftlicher Bewertung und Zertifizierung einerseits und wirtschaftlicher Verwertung andererseits konstituiert eine besondere Beziehung zwischen Wissenschaft und Verlagen, die die Marktbeziehung überformt und wechselseitige Abhängigkeiten erzeugt (Hanekop und Wittke 2006, 2007).

Für wissenschaftliche AutorInnen, besonders in den o. g. Wissenschaftsdisziplinen, haben Journale eine zentrale Bedeutung als Institution für die wissenschaftliche Bewertung und $\mathrm{Zu}$ weisung von Reputation. Die Beziehung zwischen AutorInnen und Verlagen bei Journalen ist traditionell (in den meisten Fächern) nicht marktförmig. AutorInnen liefern ihre Aufsätze kostenlos an die Journale, damit diese die Bewertung (peer review) organisieren, sie anschließend mit dem Zertifikat dieses Jour- 
nals versehen, veröffentlichen und verbreiten. Der Impaktfaktor des Journals wirkt als formeller Indikator für die Güte des Zertifikates. Gleichzeitig bildet sich in der fachspezifischen $\mathrm{Pu}-$ blikationskultur der jeweiligen Scientific Community ein allgemein anerkanntes Ranking fachbezogener Journale, an dem sich die wissenschaftliche Anerkennung und Reputation von AutorInnen in der Community orientiert. Reputation ist die Währung im Wissenschaftssystem, anhand der über die Verteilung von Forschungsmitteln, wissenschaftlichen Karrieren und Aufstiegschancen entschieden wird. Wissenschaftliche AutorInnen sind darauf angewiesen zu publizieren und daran interessiert, ihre Aufsätze in einem möglichst hochrangigen Journal unterzubringen. In diesem Prozess der Bewertung und Reputationsverteilung zwischen AutorInnen und Journalen spielt Geld (bisher) keine Rolle. Diese nicht-monetäre Beziehung entspricht den Prinzipien der akademischen Wissensproduktion, weil sie gewährleistet, dass die Bewertung und Verteilung von Reputation nicht durch monetäre Hürden oder Einflüsse beeinträchtigt wird.

Allerdings führte diese Auslagerung der wissenschaftlichen Bewertung an Verlage dazu, dass die in Journalen publizierten wissenschaftlichen Ergebnisse kein öffentliches Gut mehr sind, sondern Waren, die durch wissenschaftliche Bibliotheken erworben und zugänglich gemacht werden müssen. Um einen kostenfreien Zugangsweg zu schaffen, bauten die Protagonisten der Green-Road-Archive, Repositorien und Webseiten auf, über die AutorInnen ihre Forschungsergebnisse verbreiten können. Ein Problem der Green Road ist, dass keine Alternativen zur Bewertungs- und Reputationsfunktion der Journale etabliert wurden, sodass AutorInnen ihre Aufsätze hierfür weiterhin in einem Journal einreichen müssen. Daher ist der grüne Weg

\section{APCs für AutorInnen führen dazu, dass ihr Zugang zu Publikations- möglichkeiten von Geld abhängt.}

in der Wissenschaft ein alternativer Zugangsweg, aber kein Ersatz für die Journale. Da Verlage weitreichende Verwertungsrechte fordern, führt dies zu einem Interessenkonflikt für AutorInnen, die zwar auch ein Interesse an kostenfreier Verbreitung haben, vor allem aber das Interesse, in einem möglichst hochrangigen Journal zu publizieren, das ihnen im Wissenschaftssystem Reputation und eine berufliche Perspektive verschafft. Dieses Spannungsverhältnis entfällt bei Gold-Open-Access-Journalen. Sie bieten alle Funktionen herkömmlicher Journale. Wenn diese außerhalb der Wissenschaft durch Verlage erstellt werden, müssen sie durch ein Geschäftsmodell finanzierbar sein. Dies geschieht durch Publikationsgebühren - die Article Processing Charges (APC) - die üblicherweise die Autoren zahlen müssen. Dies führt dazu, dass die Publikation in solchen Open-AccessJournalen für Autoren nicht kostenfrei ist, sondern der Zugang zur Publikation von den finanziellen Möglichkeiten der Autoren bzw. ihrer Arbeitgeber, d. h. von Geld abhängt. Gold Open Access steht daher ebenfalls in einem Spannungsverhältnis zu den normativen Prinzipien der Wissenschaft. Daher ist es für Gold-Open-Access-Journale wichtig, dass die Gebühren bezahlbar bleiben und die Wissenschaftsorganisationen sie für die AutorInnen finanzieren. Andernfalls können Publikationsgebühren dazu führen, dass AutorInnen oder wissenschaftliche Communities das Open-Access-Publikationsmodell mit Publikationsgebühren nicht akzeptieren und für sie kostenfreie Journale oder Publikationsformen präferieren, wenn sie gleichwertige Alternativen haben (Taubert 2014, 2017). Diese Problematik verschärft sich bei einer generellen Umstellung auf Open-Access-Publikationsgebühren, in der diese Alternativen entfallen.

\section{Die bisherige Entwicklung von Open Access}

Open Access ist trotz der wachsenden Unterstützung, insbesondere durch die Wissenschaftsorganisationen, hinter den Erwartungen zurückgeblieben. Dies betrifft insbesondere Repositorien der Green Road, aber teilweise auch Gold-Open-Access-Journale. Der Verbreitungsgrad von Archiven und Repositorien der Green Road und der Gold-Open-Access-Journale liegt jeweils zwischen 15-18 \% (RIN 2015; Björk et al. 2014). Stärker gewachsen sind in den letzten Jahren Aufsätze, die von AutorInnen in sozialen Netzwerken und auf anderen Webseiten hochgeladen werden. Die Schätzungen gehen hier weit auseinander, die RIN-Studie schätzt $9 \%$, Björk über $30 \%$ (Björk 2016, S. 132). Nimmt man noch ,illegale“ Server, wie z. B. Sci-Hub, hinzu, kommt man auf $50 \%$ oder mehr (Björk 2017). Die Verbreitung von OA-Archiven und Repositorien stagniert seit einigen Jahren. AutorInnen stellen ihre Aufsätze eher selten in Repositorien ein, auch dann nicht, wenn sie dazu aufgefordert werden (Fry und Probets 2011) oder ein Mandat ihrer Forschungseinrichtung sie dazu verpflichtet. Anders ist die Situation in Fächern, in denen es große disziplinspezifische Repositorien gibt (z. B. ArXive, Pubmed central, SSRN). Auch in sozialen Netzwerken wie Researchgate und Mendely sind AutorInnen zunehmend bereit, Aufsätze verfügbar zu machen. Sie sind der am stärksten wachsende Bereich von Green Open Access (Björk 2017). Demgegenüber hat die Verbreitung der Gold-Open-Access-Journale zugenommen. Insbesondere haben die kommerziellen Verlage an Bedeutung gewonnen. Das Feld der Verlage, die Gold-Open-Access-Journale herausgeben, ist sehr heterogen. Auf der einen Seite gibt es wissenschaftsnahe, meist nichtkommerzielle Verlage. Die neuen Open-Access-Verlage kommen oft aus der Open-Access-Bewegung (z. B. PLOS) oder sie sind, wie Hindawi oder Copernicus, erklärte Dienstleister für die Wissenschaft mit vergleichsweise günstigen APCs. Außerdem gehören in diese Gruppe die scholarly publisher, professionelle Verlage, die wissenschaftlichen Fachgemeinschaften (z. B. IOP Publishing) oder Wissenschaftsorganisationen (z. B. Oxford University Press) gehören. Ihr Ge- 
schäftsmodell ist primär lizenzbasiert, sie haben aber früh begonnen, Journale in OA-Journale umzuwandeln und auch neue OA-Journale zu gründen (Laakso et al. 2016).

Auf der anderen Seite nutzen auch kommerzielle Verlage zunehmend das Geschäftsmodell von Gold-Open-Acess-Journalen mit APCs, soweit dies eine geeignete Strategie zur Verfolgung ihrer wirtschaftlichen Interessen darstellt bzw. von einflussreichen Wissenschaftsorganisationen gefordert wird (z. B. NIH, Wellcome Trust, EU). Sie haben OA-Verlage gekauft (wie Springer Biomed Central mit 200 OA-Journalen) oder in den letzten Jahren auch zunehmend Journale in OA umgewandelt bzw. neue OA-Journale gegründet (Laakso et al. 2016). Ihre Publikations- ist damit gemeint? Aufgrund der wachsenden Konzentration unter wissenschaftlichen Verlagen erscheinen über $50 \%$ der Aufsätze in den Naturwissenschaften bei den fünf größten Verlagen: Elsevier, Springer, Wiley, Tayler \& Francis, American Chemical Society (Larivière et al. 2015). Nationale und internationale Allianzen der Wissenschaftsorganisationen wollen in den regelmäßigen Lizenzverhandlungen mit Verlagen durchsetzen, dass deren Journale bis 2020 in Open-Access-Journale umgewandelt werden (Meijer 2015; Schimmer et al. 2015; Schimmer 2017). Das Ziel von Open Access wäre verwirklicht, allerdings mit Publikationskosten, die kaum unter den gegenwärtigen Lizenzkosten liegen dürften, da die bisherigen Lizenzkosten schrittweise

\title{
Ein zentrales Unterscheidungskriterium ist, ob die APCs pauschal
}

\author{
von den Wissenschaftsorganisationen an den Verlag gezahlt werden sollen, \\ oder ob AutorInnen individuell APCs für Artikel aufbringen müssen.
}

gebühren richten sich am Markt aus. Zusätzlich haben sie das sogenannte hybride Geschäftsmodell eingeführt, bei dem einzelne Artikel durch Zahlung einer Publikationsgebühr für OA freigekauft werden können. Die RIN-Studie hebt hervor, dass mittlerweile zwei Drittel der lizenzpflichtigen Journale hybride Zugangsmöglichkeiten bieten (RIN 2015). Der mit 2,4\% deutlich geringere Anteil der OA-Aufsätze in hybriden Journalen weist allerdings darauf hin, dass bisher nur wenige AutorInnen davon Gebrauch machen.

Die Publikationsgebühr der meisten OA-Journale schwankt zwischen 1000 und 1800 Euro (Schimmer et al. 2015). Sie korreliert mit dem Impaktfaktor der Journale, wie Romeu et al. (2014) in einer Untersuchung mit 2617 Open-Access-Journalen zeigen konnten. Je höher der Impaktfaktor des Journals, umso höher ist meist die Publikationsgebühr. Die APC für die hybriden Journale liegt zwischen 2000 und 3000 Euro. Die APC sind i. d. R. von den AutorInnen zu zahlen, die sich um die Übernahme durch ihre Forschungseinrichtung, einen Publikationsfonds oder die Forschungsförderung bemühen müssen (Fournier 2013).

In dieser Situation haben sich führende Wissenschaftsorganisationen in der oa2020-Initiative mit dem Ziel zusammengeschlossen, in den kommenden Jahren das lizenzbasierte Geschäftsmodell der Journale in ein Open-Access-Modell zu transformieren.

\section{Die Umwandlung in Gold Open Access mit Publikationsgebühren}

Die Umwandlung in Open-Access-Journale mit Publikationsgebühren ist keine neue Entwicklung, aber die Strategie der Wissenschaftsorganisationen zielt auf eine neue Dimension - es geht um die large-scale transition bis 2020 (oa2020 2016). Was in Publikationskosten umgewandelt werden. Sie schließen auch die Flaggschiffe der marktführenden Verlage ein, den Kern ihrer Wertschöpfung. Allerdings gehen die Wissenschaftsorganisationen auf der Basis von Schätzungen davon aus, dass die umfassende Umstellung auf Open-Access-Journale mittelfristig zur Begrenzung der Ausgabensteigerung führen wird (RIN 2015; Schimmer et al. 2015). Die Umstellung auf Publikationsgebühren würde die Verhandlungsposition der Wissenschaft verbessern, so die Erwartung (Ad-hoc-AG Open-Access-Gold 2016).

Um Risiken einer generellen Umstellung auf Publikationsgebühren für die AutorInnen einzuschätzen, werden im Folgenden die Zahlungsmodalitäten betrachtet, über die verhandelt wird. Interessant ist insbesondere, ob und in welcher Weise AutorInnen betroffen sind. Ein zentrales Unterscheidungskriterium ist daher, ob die APCs pauschal von den Wissenschaftsorganisationen an den Verlag gezahlt werden sollen, oder ob es (wie bisher bei Gold-Open-Acess-Journalen) individuelle APCs für Artikel geben soll. Grundsätzlich gibt es drei Szenarien: (1) die Umwandlung in Open-Access-Journale mit pauschaler Publikationsgebühr; (2) die Umwandlung in hybride Journale mit pauschaler Publikationsgebühr; (3) individuelle APCs für OA-Aufsätze, die mit den Lizenzgebühren der Forschungseinrichtung verrechnet werden.

Ein Beispiel für das erste Szenario ist das Projekt SCOAP3, in dem schrittweise alle für die Hochenergiephysik relevanten Journale auf Gold Open Access umgestellt werden. Federführend in diesem breiten Konsortium, dem Fachgesellschaften, Bibliotheken und Förderorganisationen angehören, ist das CERN. Die Publikationsgebühren werden durch Umschichtung der bisherigen Lizenzgebühren finanziert (Mele 2017; Romeu et al. 2014). Hier profitieren alle WissenschaftlerInnen, die erfolgreich einen Aufsatz in einem der Journale eingereicht haben. Unter diesen Bedingungen sind keine Risiken für Wissenschaftler- 
Innen erkennbar, Zugang und Publikation sind für LeserInnen und AutorInnen kostenfrei.

Ein Beispiel für das zweite Szenario ist der 2016 abgeschlossene Vertrag zwischen der Vereinigung der Niederländischen Universitäten (VSNU) und dem Verlag der American Chemical Society (ACS). Er sieht vor, dass alle in deren Journalen veröffentlichten Aufsätze von niederländischen WissenschaftlerInnen als Open Access verfügbar werden (Meijer 2017). Die AutorInnen können publizieren wie bisher, haben aber zusätzlich den Vorteil, dass ihr Aufsatz kostenfrei gelesen werden kann. Die vollständige Umwandlung der Journale in Gold Open Access ist nicht von dem Publikationsverhalten der WissenschaftlerInnen abhängig. Dieses Modell scheint allerdings gegenüber kommerziellen Verlagen schwer durchsetzbar, im obigen Beispiel handelt es sich um einen scholarly publisher.

Ein Beispiel für das dritte Szenario ist der Vertrag des Austrian Academic Consortium mit dem scholarly publisher IOP, in dem die von WissenschaftlerInnen gezahlten APCs mit den Lizenzgebühren des Konsortiums verrechnet werden. Diese Gegenrechnung (Offsetting) ist für die Beteiligten aus der Wissenschaft aufwendig und unbefriedigend (Hall und Kromp 2017). Die APCs werden den Autoren erstattet, aber diese entscheiden über die OA-Veröffentlichung. Hier müssen folgende Risiken bedacht werden: (1) AutorInnen könnten sich gegen die Open Access Publikation ihres Artikels entscheiden, wenn dies nicht ihrem Publikationsverhalten und der Publikationskultur ihrer Community entspricht, es Ihnen zu aufwendig oder das Verfahren intransparent ist. Ähnliches war beim self archiving festzustellen. Zudem gibt es entsprechende Hinweise in der

\section{Da die Höhe der Publikations-} gebühren mit dem Ranking der Journale korreliert, könnten die Reputationschancen

$$
\begin{gathered}
\text { für AutorInnen durch finanzielle } \\
\text { Erwägungen behindert werden. }
\end{gathered}
$$

Studie von Taubert aus dem Bereich der Mathematik und Astronomie (Taubert 2014, 2017). Im Kontext der Umwandlungsstrategie hätte dies zur Folge, dass diese auf niedrigem Niveau stattfindet oder insgesamt gefährdet ist. (2) Da hybride Journale auch gebührenfreie Publikationsmöglichkeiten bieten, ist das Risiko zunächst begrenzt. Nach der generellen Umwandlung in Gold-Open-Access-Journale entfällt diese Wahlmöglichkeit, sodass AutorInnen und Wissenschaftseinrichtungen mit begrenzten finanziellen Ressourcen benachteiligt wären. Wichtig sind institutionalisierte, transparente Prozesse, die auch unter- schiedliche Publikationskulturen berücksichtigen. Eine empirisch basierte Evaluation wäre sinnvoll. (3) Da die Höhe der Publikationsgebühren mit dem Ranking der Journale korreliert (s. o.), könnte dies dazu führen, dass Reputationschancen durch finanzielle Erwägungen behindert werden und es für AutorInnen schwerer wird, in relevanten, hochgerankten Journalen zu publizieren.

\section{Fazit}

Nationale und internationale Allianzen von Wissenschaftsorganisationen wollen Open Access in den Lizenzverhandlungen mit den Verlagen durch eine Umwandlung der Geschäftsmodelle erreichen. Statt des Zugangs zu Journalen soll die Publikation in Journalen kostenpflichtig werden, mit Risiken für die offene, ungehinderte Publikation neuer wissenschaftlicher Erkenntnisse. Gegenwärtig sind drei Lösungsansätze in der Diskussion: Erstens, das Risiko finanzieller Hürden für die Publikation wird durch die pauschale Zahlung von Publikationsgebühren durch Wissenschaftsorganisationen vermieden. Das zentrale Problem wird darin bestehen, sie gegenüber den marktführenden kommerziellen Verlagen durchzusetzen. Zweitens, das Risiko wird zunächst durch die individuelle Wahlmöglichkeit der AutorInnen zwischen Open Access oder kostenfreier Publikation umgangen. Auch wenn diese Risiken im Rahmen von hybriden Umstellungsszenarien noch umgangen werden können, sollten bereits angemessene Lösungen und Prozesse entwickelt (Ad-hoc-AG Open-Access-Gold 2016) und in den Publikationsstrategien der WissenschaftlerInnen verankert werden. Drittens, individuelle Publikationsgebühren für AutorInnen, die durch deren Einrichtung oder eine Förderorganisationen im Rahmen von OffsettingVerträgen erstattet werden, könnten zur Einschränkung von Publikations- und Reputationschancen führen, wenn die Finanzierung nicht für alle AutorInnen sichergestellt oder die Abwicklung nicht autorenfreundlich organisiert wird. Zumal sie sich bei der Wahl der Publikationsform meist an gewohnten Publikationsstrategien und der Publikationskultur ihrer Community orientieren. Hier wäre eine empirische Evaluation der konkreten Regelungen und Prozesse sinnvoll. Umwandlungsszenarien mit individuellen Publikationsgebühren im Rahmen von Offsetting-Verträgen haben womöglich ein weiteres Risiko: Sie könnten ihr Ziel verfehlen, wenn AutorInnen und WissenschaftlerInnen APCs vermeiden und sich gegen Open Access entscheiden.

\footnotetext{
Literatur

Ad-hoc-AG Open-Access-Gold (2016): Empfehlungen zur Open-Access-Trans-

formation der Schwerpunktinitiative „Digitale Information“ der Allianz der

deutschen Wissenschaftsorganisationen. Online verfügbar unter

https://doi.org/10.3249/allianzoa.011, zuletzt geprüft am 12.06.2017.

Björk, Bo-Christer (2016): The Open Access Movement at a Crossroads.

Are the Big Publishers and Academic Social Media Taking Over? In: Learned Publishing 29 (2), S. 131-134. Online verfügbar unter https://doi.org/10.1002/ leap.1021, zuletzt geprüft am 12.06.2017.
} 
Björk, Bo-Christer (2017): Gold, Green, and Black Open Access. In: Learned Publishing 30 (1), S. 173-175. Online verfügbar unter https://doi.org/10.1002/ leap.1096, zuletzt geprüft am 12.06.2017.

Björk, Bo-Christer; Laakso, Mikael; Welling, Patrick; Paetau, Patric (2014): Anatomy of Green Open Access. Journal of the Association for Information Science and Technology 65 (2), S. 237-250. Online verfügbar unter https://doi.org/10.1002/asi.22963, zuletzt geprüft am 12.06.2017.

Crawford, Walt (2016): Gold Open Access Journals 2011-2015. Livermore, CA. Online verfügbar unter http://waltcrawford.name/goaj1115.pdf, zuletzt geprüft am 12.06.2017.

Fournier, Johannes; Weihberg, Roland (2013): Das Förderprogramm „Open Access Publizieren“ der DFG. In: Zeitschrift für Bibliothekswesen und Bibliographie 60 (5), S. 236-243. Online verfügbar unter http://www.dfg.de/download/ pdf/foerderung/programme/lis/130522_fournier_weihberg_dfg_ foerderprogramm_oap.pdf, zuletzt geprüft am 12.06.2017.

Fry, Jenny; Probets, Steve (2011): PEER Behavioural Research - a Final Report. Online verfügbar unter http://www.peerproject.eu/fileadmin/media/reports/ PEER_D4_final_report_29SEPT11.pdf, zuletzt geprüft am 12.06.2017.

Gläser, Jochen (2006): Wissenschaftliche Produktionsgemeinschaften: Die soziale Ordnung der Forschung. Frankfurt am Main: Campus.

Hall, Steven; Kromp, Brigitte (2017) Two Perspectives on Offsetting from One of the Earliest Experiments: Austria and IOP Publishing. Vortrag auf der 13. Berlin Open Access Konferenz am 22. 03.2017. Online verfügbar unter https://oa2020.org/wp-content/uploads/mp4s/B13_Steven_Hall_Brigitte_ Kromp.mp4, zuletzt geprüft am 12.06.2017.

Hanekop, Heidemarie (2014): Wandel des wissenschaftlichen Publikationssystems durch das www? Die Wirkung wissenschaftlicher Suchportale und Suchmaschinen. In: kommunikation@gesellschaft 15, S.1-28. Online verfügbar unter http://nbn-resolving.de/urn:nbn:de:0168-ssoar-378492, zuletzt geprüft am 12.06.2017.

Hanekop, Heidemarie; Wittke, Volker (2006): Das wissenschaftliche Journal und seine möglichen Alternativen: Veränderungen der Wissenschaftskommunikation durch das Internet. In: Svenja Hagenhoff (Hg.): Internetökonomie in der Medienbranche. Göttingen: Universitätsverlag Göttingen, S. 201-234.

Hanekop, Heidemarie; Wittke, Volker (2007): Der Einfluss des Internets auf die Rekonfiguration des Systems wissenschaftlichen Publizierens. In: Ulrich Dolata und Raimund Werle (Hg.): Gesellschaft und die Macht der Technik: Sozioökonomischer und institutioneller Wandel durch Technisierung. Frankfurt am Main: Campus, S. 201-220.

Hanekop, Heidemarie; Wittke, Volker (2013): Wandel des wissenschaftlichen Publikationssystems durch das Internet - Sektorale Transformation im Kontext institutioneller Re-Konfiguration. In: Ulrich Dolata und Jan-Felix Schrape (Hg.): Internet, Mobile Devices und die Transformation der Medien. Berlin: edition sigma, S. 147-176.

Hofmann, Janette (2014): Open Access: Ein Lackmustest. Online verfügbar unter http://ssrn.com/abstract=2515844, zuletzt geprüft am 12.06.2017.

Laakso, Mikael; Solomon, David; Björk, Bo-Christer (2016): How Subscriptionbased Scholarly Journals Can Convert to Open Access: A Review of Approaches. In: Learned Publishing 29 (4), S. 259-269.

Larivière, Vincent; Haustein, Stefanie; Mongeon, Phillippe (2015): The Oligopoly of Academic Publishers in the Digital Era. In: PLOS ONE 10 (6), S. 1-15, e0127502. Online verfügbar unter https://doi.org/10.1371/journal.pone.0127502, zuletzt geprüft am 12.06.2017.
Meijer, Gerard (2017): Open Access to Scientific Publications (in The Netherlands). Vortrag auf der 13. Berlin Open Access Konferenz am 22. 03.2017. Online verfügbar unter https://oa2020.org/wp-content/uploads/pdfs/B13_Gerard_ Meijer.pdf, zuletzt geprüft am 12.06.2017.

Mele, Salvatore (2017): Concepts of Open Access Transformation: The SCOAP3 Experience. Vortrag auf der 13. Berlin Open Access Konferenz am 22.03.2017. Online verfügbar unter https://oa2020.org/wp-content/uploads/mp4s/B13_ Salvatore_Mele.mp4, zuletzt geprüft am 29.03.2017.

Merton, Robert (1985): Entwicklung und Wandel von Forschungsinteressen. Aufsätze zur Wissenschaftssoziologie. Frankfurt am Main: Suhrkamp.

oa2020 (2016): Expression of Interest in the Large-scale Implementation of Open Access to Scholarly Journals. Online verfügbar unter https://oa2020.org/ mission, zuletzt geprüft am 12.06.2017.

RIN - Research Information Network (2015): Monitoring the Transition to Open Access. A Report for the Universities UK, Open Access Co-ordination Group, August 2015. Online verfügbar unter http://www.universitiesuk.ac.uk/policyand-analysis/reports/Documents/2015/monitoring-the-transition-to-openaccess.pdf, zuletzt geprüft am 12.06.2017.

Romeu, Clément; Gentil-Beccot, Anne; Mansuy, Anne; Mele, Salvatore; Vesper, Martin (2014): The SCOAP3 Initiative and the Open access Article-processingcharge Market. Global Partnership and Competition Improve Value in the Dissemination of Science. Online verfügbar unter http://www.scoap3.de/ fileadmin/dateien/Dokumente/SCOAP3-APC.pdf, zuletzt geprüft am 12.06.2017.

Schimmer, Ralf (2017): OA2020: Progress and Outlook. Towards a NCP Network and Governance Structure: OA2020 proposal. Online verfügbar unter https://oa2020.org/wp-content/uploads/pdfs/B13_Ralf_Schimmer_Talk2.pdf, zuletzt geprüft am 12.06.2017.

Schimmer, Ralf; Geschuhn, Kai Karin; Vogler, Andreas (2015): Disrupting the Subscription Journals' Business Model for the Necessary Large-scale Transformation to Open Access. Online verfügbar unter https://dx.doi.org/10.17617/ 1.3, zuletzt geprüft am 12.06.2017.

Simukovic, Elena (2016): Open Access - the Better Access? Academic Publishing and Its Politics. $15^{\text {th }}$ Annual STS Conference Graz 2016. Online verfügbar unter https://www.researchgate.net/publication/301831970_0pen_Access_-_the_ better_access_Academic_publishing_and_its_politics, zuletzt geprüft am 12.06.2017.

Taubert, Niels (2014): Green Open Access in Mathematik und Astronomie. In: Heinrich Parthey und Walther Umstätter (Hg.): Forschung und Publikation in der Wissenschaft. Wissenschaftsforschung Jahrbuch 2013. Berlin: Wissenschaftlicher Verlag.

Taubert, Niels (2017): Fremde Galaxien und abstrakte Welten. Open Access in Astronomie und Mathematik. Im Erscheinen.

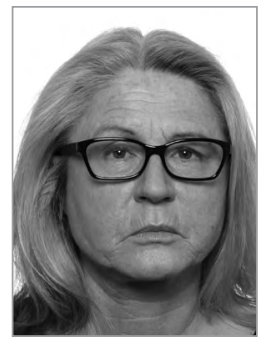

\section{HEIDEMARIE HANEKOP}

ist Soziologin und arbeitet als wissenschaftliche Mitarbeiterin am Soziologischen Forschungsinstitut Göttingen in zahlreichen interdisziplinären Forschungsprojekten in der Technik-, Wissenschaftsund Innovationssoziologie. U. a. Internetforschung, Wissenschaftskommunikation, webbasierte Dienstleistungen, kollaborative Innovationen. 